\title{
First Principles Semiclassical Calculations of Vibrational Eigenfunctions
}

\section{Citation}

Ceotto, Michele, Stéphanie Valleau, Gian Franco Tantardini, and Alán Aspuru-Guzik. 2011. First principles semiclassical calculations of vibrational eigenfunctions. Journal of Chemical Physics 134(23), 234103.

\section{Published Version}

doi:10.1063/1.3599469

\section{Permanent link}

http://nrs.harvard.edu/urn-3:HUL.InstRepos:8404289

\section{Terms of Use}

This article was downloaded from Harvard University's DASH repository, and is made available under the terms and conditions applicable to Open Access Policy Articles, as set forth at http:// nrs.harvard.edu/urn-3:HUL.InstRepos:dash.current.terms-of-use\#OAP

\section{Share Your Story}

The Harvard community has made this article openly available.

Please share how this access benefits you. Submit a story.

Accessibility 


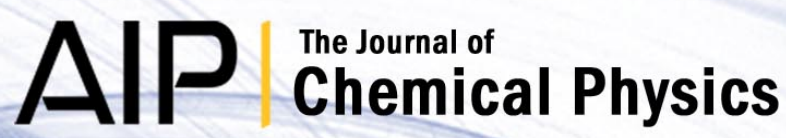

\section{First principles semiclassical calculations of vibrational eigenfunctions}

Michele Ceotto, Stéphanie Valleau, Gian Franco Tantardini, and Alán Aspuru-Guzik

Citation: J. Chem. Phys. 134, 234103 (2011); doi: 10.1063/1.3599469

View online: http://dx.doi.org/10.1063/1.3599469

View Table of Contents: http://jcp.aip.org/resource/1/JCPSA6/v134/i23

Published by the American Institute of Physics.

\section{Additional information on J. Chem. Phys.}

Journal Homepage: http://jcp.aip.org/

Journal Information: http://jcp.aip.org/about/about_the_journal

Top downloads: http://jcp.aip.org/features/most_downloaded

Information for Authors: http://jcp.aip.org/authors

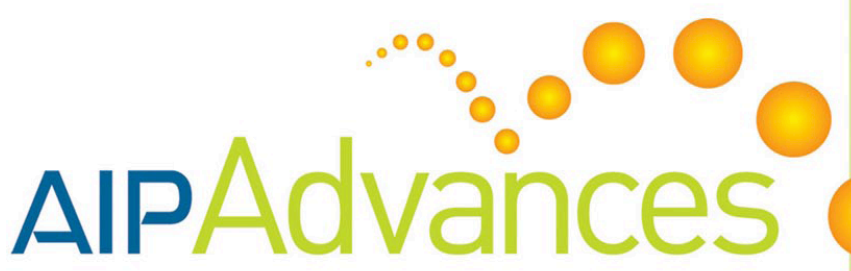

Explore AIP's new open-access journal

Article-level metrics now available

Join the conversation!

Submit Now 


\title{
First principles semiclassical calculations of vibrational eigenfunctions
}

\author{
Michele Ceotto, ${ }^{1, \text { a) }}$ Stéphanie Valleau, ${ }^{1,2}$ Gian Franco Tantardini, ${ }^{1,3}$ \\ and Alán Aspuru-Guzik ${ }^{2}$ \\ ${ }^{1}$ Dipartimento di Chimica Fisica ed Elettrochimica, Università degli Studi di Milano, via Golgi 19, \\ 20133 Milano, Italy \\ ${ }^{2}$ Department of Chemistry and Chemical Biology, Harvard University, 12 Oxford Street, Cambridge, \\ Massachusetts 02138, USA \\ ${ }^{3}$ Istituto CNR di Scienze e Tecnologie Molecolari, via Golgi 19, 20133 Milano, Italy
}

(Received 24 March 2011; accepted 22 May 2011; published online 17 June 2011)

\begin{abstract}
Vibrational eigenfunctions are calculated on-the-fly using semiclassical methods in conjunction with $a b$ initio density functional theory classical trajectories. Various semiclassical approximations based on the time-dependent representation of the eigenfunctions are tested on an analytical potential describing the chemisorption of $\mathrm{CO}$ on $\mathrm{Cu}(100)$. Then, first principles semiclassical vibrational eigenfunctions are calculated for the $\mathrm{CO}_{2}$ molecule and its accuracy evaluated. The multiple coherent states initial value representations semiclassical method recently developed by us has shown with only six $a b$ initio trajectories to evaluate eigenvalues and eigenfunctions at the accuracy level of thousands trajectory semiclassical initial value representation simulations. () 2011 American Institute of Physics. [doi:10.1063/1.3599469]
\end{abstract}

\section{INTRODUCTION}

Quantum properties of molecular systems are encoded in the eigenvalues and eigenfunctions of the molecular Hamiltonian. Many fundamental properties of molecules can be inferred from features such as their eigenfunctions' nodes and their eigenvalue spectra. For example, the nodal shapes for higher vibrational eigenstates in a normal mode set of coordinates reveal whether and how the modes are coupled, i.e., if the coupling is resonant. Furthermore, by comparing the nodes of the wave function to the histories of ensembles of classical trajectories, one can understand the different types of resonances between eigenmodes and gain insight about the nature of these resonances, for example, how "classical" these resonances are. Unfortunately exact quantum mechanical methods for computing eigenfunctions and eigenvalues are limited to systems comprising a few degrees of freedom and most of the methods rely on having a precomputed analytical potential energy surface (PES). ${ }^{1-4}$ The computation of PES is usually a trade-off between human effort and accuracy. This is especially true when one is dealing with complex or floppy systems or bond-breaking processes. On the other hand, while one can employ classical methods to study large many-particle systems, these methods are unable to reproduce intrinsically quantum features: for example, they cannot predict the form of the quantum eigenfunctions. For these reasons, a desirable method should not depend on the analytical development of PES but nevertheless provide access to quantum properties, ${ }^{5-7}$ such as the vibrational eigenfunctions. First-principles molecular dynamics (FPMD) approaches have evolved as an alternative to the pre-computation of PES and these allow for the inclusion of

\footnotetext{
a)Electronic mail: michele.ceotto@unimi.it.
}

quantum effects in the nuclear dynamics. ${ }^{8-17}$ Although there has been great development in this field, to our knowledge, calculations of molecular eigenfunctions using on-the-fly approaches have not been explored.

In order to reach this goal, several semiclassical approximations to quantum nuclear dynamics will be adopted. An appealing semiclassical method for the computation of eigenfunctions should be able to provide accurate quantized solutions for polyatomic systems and be easy to apply, preferably in a black-box fashion. Furthermore, as mentioned before, if the method can be cast into an FPMD approach, one can avoid the explicit construction of the PES. Some of the original semiclassical methods were based on the Einstein-Brillouin-Keller (EBK) theorem. ${ }^{18}$ According to these methods an eigentrajectory with specific EBK quantization conditions is searched and a uniform approximation to be corrected with the Maslov phase at the caustic point discontinuity is used. ${ }^{19}$ This is equivalent to a static JeffreysWentzel-Kramers-Brillouin (JWKB)-like formalism, ${ }^{20}$ in which the task of finding the multidimensional eigentrajectories seems to be quite involved.

As an alternative to EBK and JWKB approaches, as we expose below, the spectral quantum features can be better understood in terms of the underlying dynamics, in particular within the semiclassical approximation, since periodic orbits set a correspondence between stationary states and their corresponding dynamics. For example, Heller and co-workers have shown strong evidence of this correspondence between classical motion and quantum effects even for spacings of combinations of overtones. ${ }^{21}$ In particular, in studies also pioneered by Heller, the vibrational eigenfunctions have been calculated on a model potential using a single classical trajectory. ${ }^{22}$ These calculations used Gaussian wavepackets of frozen width, which is generally, a computationally desirable feature: The Fourier transform of a simple Gaussian integrand 
following a quasi-periodic trajectory leads directly to an approximation to the eigenfunctions of the system of study. In short, semiclassical methods are based on a linear superposition of Gaussian coherent states that lie along the quantized classical trajectories. Such approach is free of any caustic problems and the agreement with the exact calculations is good in the tunneling region. In principle, one can use any classical trajectory to calculate eigenfunctions. However as will be shown below, this will affect the eigenfunctions' accuracy. Analytical considerations have shown that semiclassical methods can reproduce eigenfunctions of the two dimensional rigid rotor $^{23}$ and of a particle in a box ${ }^{24}$ exactly, while for the Morse potential eigenstates, the agreement is accurate. ${ }^{25}$ Still analytically, the three-dimensional isotropic harmonic oscillator eigenfunctions are calculated by representing the eigenfunctions as the integral of a semiclassical wavepacket over the Lagrangian manifold corresponding to the desired state. In this formulation, a parametric dependence of the wavepacket on the variable describing the Lagrangian manifold corresponding semiclassically to the state of interest is used instead of a time dependence. ${ }^{26}$ This formulation is uniform, i.e, it is free of caustic singularities, and it is semiclassical since it approaches the exact wave function uniformly for small $\hbar$ values $(\hbar \rightarrow 0)$. Exact eigenstates have been derived for several systems, including the hydrogen atom, where the semiclassical expression reproduced exactly a given electronic orbital state by integrating over the radial and angular parameters. ${ }^{27-29}$ Nevertheless, one should keep in mind that semiclassical theories remain an approximation to the full quantum mechanical picture and in general, only yield approximate eigenfunctions. To our knowledge, the only general procedure to estimate the error arising from semiclassical approximations other than a direct comparison with the exact quantum eigenfunctions, is a perturbative series correction of the propagator. $^{30-33}$

In this paper, a set of semiclassical molecular dynamics methods will be employed to calculate vibrational eigenfunctions. First, the methods will be tested on analytical potentials where exact calculations can be performed, and then coupled with a first principles approach, in which the potential energy surfaces are computed on-the-fly using density functional theory (DFT). We employ a recent implementation ${ }^{34,35}$ of the original time-averaging filtering of the semiclassical initial value representation method (SC-IVR) (Ref. 36) and extend it for the purpose of calculating vibrational eigenfunctions. This method uses a suitable set of delocalized coherent states that resembles a linear combination of the eigenfunctions of the system to reproduce quantum spectral features. In this way, the multiple coherent states SC-IVR (MC-SC-IVR) mimics the multiple vibrational components and successfully reproduce well-defined spectra for the several systems which have been tested. ${ }^{34,35,37}$ The major advantages of the method are that very few trajectories can accurately reproduce SC-IVR spectra obtained with thousands of trajectories and further that it can easily be implemented into a first principles molecular dynamics calculation. More recently Roy et al. ${ }^{38}$ also used such delocalization of coherent states for the calculation of the vibrational power spectrum of formaldehyde. This is another example of first-principles SC-IVR, as well as it is the work of Pollak and Tatchen on the absorption spectrum of the same molecule. ${ }^{17}$

In Sec. II, we will review the expression for computing the eigenfunctions using wavepackets and semiclassical approximations. In Sec. III, we discuss the computation of eigenfunctions with different methods for an analytical potential. In Sec. IV, first principles semiclassical eigenfunctions are calculated for the carbon dioxide molecule and, finally, conclusions are drawn in Sec V.

\section{SEMICLASSICAL APPROXIMATION FOR EIGENFUNCTION CALCULATIONS}

\section{A. Time-dependent formulation for eigenfunctions calculation}

Usually, the time-independent approaches to eigenfunction and eigenvalue calculation are based on the diagonalization of the Hamiltonian, which is represented in a suitable basis set. The computational cost of these types of approaches grows exponentially with the dimensionality of the system. A time-dependent representation could be a valid alternative, since it opens new avenues to quantum approximation methods. ${ }^{22}$ Following, we review the basics of the time-dependent method for computing eigenfunctions. Consider a general non-stationary state represented by the wavepacket $\Psi(\mathbf{x}, t)$ with Schroedinger dynamics,

$$
i \hbar \frac{\partial}{\partial t} \Psi(\mathbf{x}, t)=\hat{H} \Psi(\mathbf{x}, t),
$$

the time-evolved wavepacket can be expressed in terms of a superposition of eigenstates $\psi_{n}(\mathbf{x})$,

$$
\Psi(\mathbf{x}, t)=\sum_{n} c_{n}(t) \psi_{n}(\mathbf{x})=\sum_{n}\left\langle\psi_{n}(\mathbf{x}) \mid \Psi(\mathbf{x}, t)\right\rangle \psi_{n}(\mathbf{x}) .
$$

By combining Eq. (2) with Eq. (1), the time-evolution can be described by the expansion coefficients $c_{n}(t)$,

$$
\sum_{n} \frac{d}{d t} c_{n}(t) \psi_{n}(\mathbf{x})=\sum_{n} c_{n}(t) E_{n} \psi_{n}(\mathbf{x})
$$

By multiplying from the left by $\left\langle\psi_{m}(x)\right|$, one obtains the dynamical equations for the coefficients $c_{m}(t)$,

$$
\frac{d c_{m}(t)}{d t}=E_{m} c_{m}(t)
$$

or, equivalently,

$$
c_{m}(t)=e^{-i E_{m} t / \hbar} c_{m}(0) .
$$

By substituting Eq. (5) into Eq. (2), the formulation of a generic non-stationary state as the evolution in terms of (stationary) eigenstates is obtained,

$$
\Psi(\mathbf{x}, t)=\sum_{n} e^{-i E_{n} t / \hbar}\left\langle\psi_{n}(\mathbf{x}) \mid \Psi(\mathbf{x}, 0)\right\rangle \psi_{n}(\mathbf{x}) .
$$

Equation (6) is inverted by applying a Fourier transform to both sides and using the Fourier representation for Dirac's delta function in the energy domain. This results in the time-dependent representation of the $n$th eigenfunction,

$$
\psi_{n}(\mathbf{x})=\frac{1}{\left\langle\psi_{n}(\mathbf{x}) \mid \Psi(\mathbf{x}, 0)\right\rangle} \frac{1}{T} \int_{-T}^{+T} e^{i E_{n} t / \hbar} \Psi(\mathbf{x}, t) d t,
$$


where $E_{n}$ are the eigenfunction energies, and $T$ is the total time the wavepacket is propagated for. By using time inversion symmetry, Eq. (7) can be computed more conveniently since it can be written as

$$
\psi_{n}(\mathbf{x} ; T) \propto \frac{2}{T} \int_{0}^{T} d t \Re e\left(\Psi(\mathbf{x}, t) e^{i E_{n} t}\right) .
$$

By either guessing or having the eigenvalue $E_{n}$, and integrating the time-dependent wave function, the value at $\mathbf{x}$ of the eigenfunction for that given eigenvalue can be retrieved. ${ }^{39}$ As one integrates up to larger $T$, the global eigenfunction is increasingly refined. If the trajectory is quasiperiodic, it is possible to obtain sufficiently converged eigenfunctions for a reasonable value of $T$.

Equation (8) expresses that any eigenstate can be obtained from a superposition of wavepackets, i.e., the same wavepacket at different times, when integrated using a Fourier phase corresponding to its eigenvalue. The Fourier phase is essential, as it interferes with the phase of the wavepacket to project out the vibrational eigenfunction. In other words, Fourier transforming the wavepacket at peak frequencies of the power spectrum leads to constructive interference of the wavepacket with itself. Instead, if the Fourier transform is at an off-peak frequency, "the wavepacket would interfere with itself haphazardly and generally destructively." 40

\section{B. De Leon-Heller semiclassical eigenfunctions}

The original semiclassical quantization of vibrational Hamiltonians has been expressed in terms of action quantization. The Einstein-Brillouin-Keller quantization (EBK) corrected by a Maslov index is one of the seminal approaches. ${ }^{18}$ The EBK method implies searching for a trajectory whose action integrals are properly quantized. The energy of such a trajectory is the semiclassical eigenvalue. However, this approach results to be too cumbersome when a multidimensional trajectory search is needed.

The time-dependent formulation presented in Eq. (8) is suitable for implementation into a semiclassical molecular dynamics perspective. As shown by Heller and Davis, ${ }^{41}$ it is convenient to replace a trajectory that quantizes the system with a linear superposition of Gaussian coherent states that are generated from that trajectory. This strategy is suggested by Eq. (8) itself, where the eigenfunction is a convolution of a time evolved wavepacket. In our simulations, the wavepacket is composed of a coherent state part,

$$
\begin{aligned}
\langle\mathbf{x} \mid \mathbf{p}(t), \mathbf{q}(t)\rangle= & \prod_{j=1}^{F}\left(\frac{\gamma_{j}}{\pi}\right)^{1 / 4} \exp \left[-\frac{\gamma_{j}}{2}\left(x_{j}-q_{j}(t)\right)^{2}\right. \\
& \left.+i p_{j}(t)\left(x_{j}-q_{j}(t)\right)\right],
\end{aligned}
$$

where $F$ is the number of degrees of freedom, $q_{j}(t)$ and $p_{j}(t)$ are the position and momentum of the classical trajectory for the $j$ th dimension, and $\gamma_{j}$ are the coherent state widths usually chosen to match the widths of the harmonic oscillator approximation to the wave function at the global minimum. The term in Eq. (9) is multiplied by a time-dependent phase term $e^{i \gamma(t) / \hbar}$ to give the semiclassical representation of the wavepacket. The phase term is crucial for the coherent buildup of the eigenfunction and it has the following form: ${ }^{41}$

$$
\gamma(t)=\int_{0}^{\mathbf{q}(t)} \mathbf{p} \cdot d \mathbf{q}-E \cdot t+\frac{T}{2} \sum_{j=1}^{F} \omega_{j}
$$

where the first two terms are the classical action, a common ingredient in every semiclassical approximation. The timeevolved wavepacket is easily obtained by generating the phase space Gaussian center's coordinates $(\mathbf{p}(t), \mathbf{q}(t))$ according to Newton's equations, the classical action and the phase $\gamma(t)$ as in Eq. (10). Initial conditions are taken from the harmonic approximation. The frequency terms in Eq. (10) resemble the semiclassical Maslov phase correction. The frequency values $\omega_{j}$ were estimated by taking a multidimensional trajectory long enough to close on itself in a time $T_{c}$ and by counting the total number of cycles $\left(M_{j}\right)$ that this trajectory makes for each degree of freedom, as originally suggested by Heller and co-workers. ${ }^{41}$ Then, the expression for the frequency is

$$
\omega_{j}=\frac{2 \pi}{T_{c}} M_{j} .
$$

The criteria for trajectory closure was set so strict that it closes only once within the total simulation time. The frequencies obtained were comparable to the harmonic estimate coming from the Hessian diagonalization at the global minimum. A suitable simulation time and trajectory closure criteria are very important since the correct phase calculation is crucial for the coherent superposition. The trajectory used to generate the coherent state should be taken long enough to adequately explore the entire manifold on which it lies. We found this semiclassical recipe for calculating the eigenfunctions simple and practical. It is, however, limited to a single classical trajectory.

\section{SC-IVR eigenfunctions}

In the same spirit as the above discussion, one can formulate the SC-IVR expression for eigenfunction calculation by invoking the Heller-Herman-Kluk-Kay (HHKK) propagator. In the SC-IVR method, ${ }^{36,42-49}$ the propagator in $F$ dimensions is approximated by the phase space integral,

$$
\begin{aligned}
e^{-i \hat{H} t / \hbar}= & \frac{1}{(2 \pi \hbar)^{F}} \int d \mathbf{p}(0) \int d \mathbf{q}(0) C_{t}(\mathbf{p}(0), \mathbf{q}(0)) \\
& e^{i S_{t}(\mathbf{p}(0), \mathbf{q}(0)) / \hbar}|\mathbf{p}(t), \mathbf{q}(t)\rangle\langle\mathbf{p}(0), \mathbf{q}(0)|
\end{aligned}
$$

where $(\mathbf{p}(t), \mathbf{q}(t))$ are the set of classically evolved phase space coordinates, $S_{t}$ is the classical action,

$$
S_{t}(\mathbf{p}(0), \mathbf{q}(0))=\int_{0}^{t} d t^{\prime}\left(\frac{\mathbf{p}^{2}\left(t^{\prime}\right)}{2 m}-V\left(\mathbf{q}\left(t^{\prime}\right)\right)\right),
$$

and $C_{t}$ is a pre-exponential factor. In the Heller-HermanKluk-Kay ${ }^{50,51}$ version of the SC-IVR, the prefactor is

$$
\begin{aligned}
C_{t} & (\mathbf{p}(0), \mathbf{q}(0)) \\
= & \sqrt{\frac{1}{2}\left|\frac{\partial \mathbf{q}(t)}{\partial \mathbf{q}(0)}+\frac{\partial \mathbf{p}(t)}{\partial \mathbf{p}(0)}-i \hbar \gamma \frac{\partial \mathbf{q}(t)}{\partial \mathbf{p}(0)}+\frac{i}{\gamma \hbar} \frac{\partial \mathbf{p}(t)}{\partial \mathbf{q}(0)}\right|}
\end{aligned}
$$


and the basis set is a product of $F$ one-dimensional coherent states of Eq. (9). For bound systems, no significant dependency on width variation was found. ${ }^{43}$ By using a $2 F \times 2 F$ symplectic (monodromy) matrix $\mathbf{M}(t)$ $\equiv\left(\partial\left(\mathbf{p}_{t}, \mathbf{q}_{t}\right) / \partial\left(\mathbf{p}_{0}, \mathbf{q}_{0}\right)\right)$, one can calculate the pre-factor of Eq. (14) from $F \times F$-sized blocks and we monitored the accuracy of the classical approximate propagation by containing the deviation of its determinant from unity to be less than $10^{-6}$. Thus, the SC-IVR representation of the time evolved wavepacket is

$$
\begin{aligned}
\Psi(\mathbf{x}, t)= & \frac{\int d \mathbf{p}(0) \int d \mathbf{q}(0)}{(2 \pi \hbar)^{F}} C_{t}(\mathbf{p}(0), \mathbf{q}(0)) e^{i S_{t}(\mathbf{p}(0), \mathbf{q}(0)) / \hbar} \\
& \langle\mathbf{x} \mid \mathbf{p}(t), \mathbf{q}(t)\rangle\langle\mathbf{p}(0), \mathbf{q}(0) \mid \mathbf{p}(0), \mathbf{q}(0)\rangle .
\end{aligned}
$$

Inserting Eq. (15) into Eq. (8), one finds the final SC-IVR expression for the eigenfunction used in this paper. The zerotime coherent-state overlap in Eq. (15) is the employed density for the Monte Carlo phase space integration. In order to gain further numerical stability for the calculation of the prefactor in Eq. (14), one can introduce a phase approximation along the lines of the separable approximation for the double times prefactor used in the time-averaging filtering. ${ }^{34,35,54}$ In this case, the prefactor at time evolution $t$ is approximated in the following manner:

$$
C_{t}(\mathbf{p}(0), \mathbf{q}(0)) \approx e^{i \phi(t) / \hbar}
$$

where $\phi(t)=$ phase $\left[C_{t}(\mathbf{p}(0), \mathbf{q}(0))\right]$. This can be a mild approximation if the equations of motion are evolved using a symplectic algorithm, since any deviation of the prefactor from its unitary module is due to numerical errors. In our simulation on analytical potentials, a fourth-order simplectic algorithm was employed. ${ }^{52}$ For the first-principles semiclassical molecular dynamics calculations, the standard velocity Verlet algorithm as implemented in the Q-CHEM package ${ }^{53}$ was used for the propagation. Note that for the case of employing Eq. (15) this approximation does not result in any additional savings of computational resources. Nevertheless, we will investigate if there are any significant differences between using the phase approximation and employing the original prefactor.

If a single classical trajectory is employed instead of a phase space integral and inserted into Eq. (8), one obtains

$$
\begin{aligned}
\psi_{n}(\mathbf{x} ; T) \propto & \frac{2 \Re e}{T} \int_{0}^{T} \frac{e^{i S_{t}(\mathbf{p}(0), \mathbf{q}(0)) / \hbar}}{(2 \pi \hbar)^{F}} e^{i E_{n} t} \\
& \times C_{t}(\mathbf{p}(0), \mathbf{q}(0))\langle\mathbf{x} \mid \mathbf{p}(0), \mathbf{q}(0)\rangle d t,
\end{aligned}
$$

which is much less computationally demanding and still different from the De Leon-Heller expression. However, both methods avoid any divergence of the semiclassical propagator at the caustic or turning point, which has been one of the major problems of the application of the WKB approximation.

\section{Multiple coherent states semiclassical initial value representation eigenfunctions}

In a typical SC-IVR simulation, the Monte Carlo phase space integration has been tested to converge with a number of trajectories of the order of thousands. ${ }^{43}$ If one requires to propagate trajectories using first-principles dynamics, this number of trajectories is prohibitive. Recently, we ${ }^{35}$ developed a method called multiple coherent states SC-IVR (MC-SC-IVR) which reduces significantly the number of classical trajectories to only a few while still preserving good accuracy. The method was developed for power spectra calculations and consists in a SC-IVR strategy that enhances as much as possible the overlap between the reference state (whose time-evolution is Fourier transformed into the power spectrum) and the exact quantum eigenfunctions. In fact, one can think of projecting the power spectrum into the phase space portrait, where it is represented by a set of multidimensional closed "eigen-trajectories" with energy equal to the peaks of the spectrum (see Fig. 1 in Ref. 35). Then, it is clear that a set of trajectories which somehow mimics this distribution is more representative than a single ground state trajectory. For this method, it was found that it is not crucial to know the exact location of the "eigen-trajectories" turning points at the values of potential energy equal to the peaks of the power spectrum. This is so because the Gaussian spreading of each coherent states lying on the top of each "eigen-trajectory" is generally wide enough to include the peaks' energy shell. In this way, with few trajectories we obtained accurate results for the $\mathrm{H}_{2} \mathrm{O}$ molecule on a model potential and for the $\mathrm{CO}_{2}$ molecule using an on-the-fly approach. ${ }^{35}$ Additionally, we obtained accurate spectra for the model potential describing the chemisorption process of $\mathrm{CO}$ on $\mathrm{Cu}(100)$ copper surface that will be used below. The agreement was excellent not only when dimensionality was reduced to the two stretching motions of a single molecule, but also when a set of four dipole coupled molecules were arranged in a monolayer fashion. ${ }^{37}$ Thus, the MC-SC-IVR method has proved to be really advantageous since the number of trajectories can be substantially reduced, ${ }^{35}$ while preserving an accuracy comparable to that obtainable with thousands of trajectory calculations. The MC-SC-IVR formulation of the eigenfunction is

$$
\begin{aligned}
\psi_{n}(\mathbf{x}) \propto & \frac{\Re e}{T} \int_{0}^{T} d t e^{i E_{n} t / \hbar} \frac{1}{(2 \pi \hbar)^{F}} \sum_{i=0}^{N_{\text {states }}-1} e^{i S_{t}\left(\mathbf{p}_{i}(0), \mathbf{q}_{i}(0)\right) / \hbar} \\
& \times C_{t}\left(\mathbf{p}_{i}(0), \mathbf{q}_{i}(0)\right)\left\langle\mathbf{p}_{i}(0), \mathbf{q}_{i}(0) \mid \mathbf{x}\right\rangle
\end{aligned}
$$

where the initial phase space conditions are the equilibrium geometry $\mathbf{q}(0)$ for the positions and the harmonic approximated momenta, namely, $p_{j, i}^{2} / 2 m=\hbar \omega_{j}(i+1 / 2)$ for each $j$ th degree of freedom of the th trajectory. Eq. (18) reduces the phase space integral to a sum over a set of $N_{\text {states }}$, which we called "eigen-trajectories," which are harmonically spaced in the energy domain. An analogous expression holds when the separable approximation is applied. In this way MC-SC-IVR includes the quantum mechanical delocalization by using a set of coherent states placed in a non-local fashion, while their centers are kept fixed during the entire simulation time. 


\section{MEASURING THE QUALITY OF THE EIGENFUNCTIONS}

The most straightforward metric for the accuracy of the semiclassical eigenfunctions, is their overlap with numerically exact solutions,

$$
O=\int\left\langle\psi_{S C}(\mathbf{x}) \mid \psi_{D V R}(\mathbf{x})\right\rangle d \mathbf{x},
$$

where $\psi_{S C}(\mathbf{x})$ is the normalized semiclassical eigenfunction calculated according to one of the approximations described above, while $\psi_{D V R}(\mathbf{x})$ is what we consider the numerically exact quantum eigenfunction calculated with the discrete variable representation method in the Sinc function basis. ${ }^{55}$ Thus, the integral of Eq. (19) becomes a sum over all Discrete Variable Representation (DVR) grid points $N_{D V R}$,

$$
O=\sum_{i=1}^{N_{D V R}} \psi_{S C}^{\star}\left(\mathbf{x}_{i}\right) \psi_{D V R}\left(\mathbf{x}_{i}\right) \Delta \mathbf{x}_{i} .
$$

However, in case of degeneracy the comparison between eigenfunctions of the same eigenvalue calculated with different methods is not so straightforward. In this case, any combination of degenerate eigenfunctions is still an eigenfunction and in principle one does not know how a set of degenerate semiclassical eigenfunctions can be matched with the exact ones. One is tempted to introduce a mixing angle that combines the eigenfunctions with sine and cosine coefficients and represent the semiclassical eigenfunctions as rotations of the exact ones, however we found this procedure to be too cumbersome when dimensionality is increased. A simpler way to overcome this impasse is on one hand, to measure to which extent the semiclassical eigenfunction is an eigenfunction of the DVR Hamiltonian matrix and on the other to measure the completeness of the basis of each group of degenerate functions within the degenerate subspace.

To reach the first goal, the following expression was introduced:

$$
\varepsilon_{1}=\frac{\left.\left|\hat{H}_{D V R}\right| \psi_{S C}\right\rangle-E_{D V R}\left|\psi_{S C}\right\rangle \mid}{E_{D V R}},
$$

where $\hat{H}_{D V R}$ is the DVR matrix representation of the Hamiltonian operator, $\left|\psi_{S C}\right\rangle$ is the semiclassical eigenfunction evaluated at the DVR grid points and $E_{D V R}$ is the exact quantum eigenvalue. In Eq. (21) the norm of the vector measuring the deviation of $\left|\psi_{S C}\right\rangle$ from being the exact ket is calculated in the numerator and weighed respect to the value of the eigenvalue. If $\left|\psi_{S C}\right\rangle$ is an eigenket, then $\varepsilon_{1}=0$. Moreover, if $\left|\psi_{S C, 1}\right\rangle$ and $\left|\psi_{S C, 2}\right\rangle$ are two degenerate precise eigenfunctions, then they should both have small values of $\varepsilon_{1}$, even if their overlap with $\left|\psi_{D V R}\right\rangle$ is arbitrary. In order to appreciate how much small $\varepsilon_{1}$ should be for an eigenfunction to be accurate, one can use Eqs. (21) together with Eq. (20) for the ground state (which is not degenerate) and then compare this with the values of $\varepsilon_{1}$ in the case of degenerate states on the same grid points.

To reach the second goal, i.e., to prove that the set of degenerate semiclassical eigenfunctions is complete within the degenerate subspace, the following quantity was introduced:

$$
\varepsilon_{2}=\sqrt{\frac{\left\langle\psi_{S C}\left|\hat{H}_{D V R}\right| \psi_{S C}\right\rangle}{E_{D V R}}},
$$

where the notation is the same as in Eq. (21). Further comments on the $\varepsilon_{2}$ parameter for a given eigenfunction $\left|\psi_{S C, i}\right\rangle$ are necessary to appreciate the meaning of Eq. (22) better. One first notes that

$$
\begin{aligned}
\varepsilon_{2, i}^{2} & =\frac{\left\langle\psi_{S C, i}\left|\hat{H}_{D V R}\right| \psi_{S C, i}\right\rangle}{E_{D V R, i}} \\
& =\frac{\sum_{l, n}\left\langle\psi_{S C, i} \mid \psi_{D V R, l}\right\rangle\left\langle\psi_{D V R, l}\left|\hat{H}_{D V R}\right| \psi_{D V R, n}\right\rangle\left\langle\psi_{D V R, n} \mid \psi_{S C, i}\right\rangle}{E_{D V R, i}},
\end{aligned}
$$

where the identity in terms of DVR complete basis set has been inserted twice. Then, by using the properties that $\left|\psi_{D V R, i}\right\rangle$ is the exact $i$ th eigenfunction of $\hat{H}_{D V R}$ of eigenvalue $E_{D V R, i}$, Eq. (24) is simplified to

$$
\varepsilon_{2, i}=\sqrt{\frac{\sum_{n}\left|\left\langle\psi_{D V R, n} \mid \psi_{S C, i}\right\rangle\right|^{2} E_{D V R, n}}{E_{D V R, i}}}
$$

For non-degenerate cases, Eq. (25) is equal to Eq. (19) plus the contributions coming from the overlap of the $i$ th semiclassical eigenfunctions with the exact eigenfunctions orthonormal to the $i$ th one. Thus, if the semiclassical approximation is really accurate, Eq. (25) should give the same value as Eq. (19) does for non-degenerate eigenvalues.
Instead, when two states $\left|\psi_{D V R, 1}\right\rangle$ and $\left|\psi_{D V R, 2}\right\rangle$ are degenerate, they can both give a significant overlap with the semiclassical eigenfunction $\left|\psi_{S C, i}\right\rangle$ which approximates one of the degenerate eigenstate. In Eq. (25), let us assume that $\left|\psi_{S C, i}\right\rangle$ is good enough to be orthogonal to all exact eigenkets which do not belong to the considered degenerate subspace. In this case, if $\left|\psi_{D V R, 1}\right\rangle$ and $\left|\psi_{D V R, 2}\right\rangle$ are two eigenfunctions that span the degenerate subspace then we can represent the semiclassical eigenfunction as follows:

$$
\left|\psi_{S C, i}\right\rangle \approx \cos \omega\left|\psi_{D V R, 1}\right\rangle+\sin \omega\left|\psi_{D V R, 2}\right\rangle,
$$

where $\omega$ is the mixing angle. By inserting this approximation for $\left|\psi_{S C, i}\right\rangle$ into Eq. (25) and considering that $E_{D V R, 1}$ $=E_{D V R, 2}=E_{D V R, i}$, one obtains $\varepsilon_{2, i}=1$. Thus, the sum of the squares of the overlaps with the semiclassical 


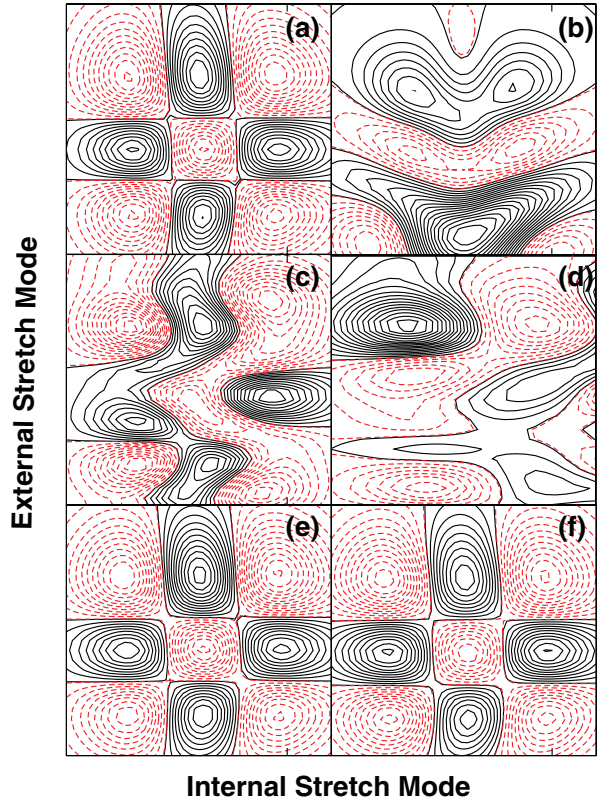

FIG. 1. Normalized vibrational eigenfunction contour plots for the $(2,2)$ eigenstate: (a) the exact DVR eigenfunction; (b) from a single ground trajectory using the semiclassical single trajectory energy estimation; (c) using De Leon-Heller method; (d) using an anharmonically corrected initial single trajectory condition; (e) from MC-SC-IVR with the approximation of Eq. (16) and eigenvalue from same level of energy calculation, i.e., MCSC-IVR with the separable approximation; (f) the same as (e) but without invoking the approximation in Eq. (16). Dashed red lines are for negative values.

eigenfunction is unitary only if the approximate eigenfunction can be written as a rotation of the exact eigenfunctions and if it is orthonormal to all others exact eigenfunctions. In other words, the closer $\varepsilon_{2}$ is to unity, the better the semiclassical eigenfunction describes that degenerate state.

\section{A. A Test Case: $\mathrm{CO}$ on $\mathrm{Cu}(100)$ Vibrational Eigenfunctions}

When a $\mathrm{CO}$ molecule adsorbs on top of a copper atom of a $\mathrm{Cu}(100)$ surface, besides the internal $\mathrm{C}-\mathrm{O}$ stretch mode, five other external modes are present. These are the two-fold frustrated rotations, the two-fold degenerate frustrated translations and the external $\mathrm{C}-\mathrm{Cu}$ stretching respect to the surface. For a pictorial representation the reader can refer to Fig. 1 in Ref. 37 for example. The analytical potential developed by Tully and co-workers ${ }^{56-58}$ has been widely used to perform theoretical and molecular dynamics simulations for this system. The potential form is

$$
V\left(\mathbf{r}_{\mathrm{C}}, \mathbf{r}_{\mathrm{O}}\right)=\sum_{i=1}^{N_{\text {copper }}} V_{i}\left(\mathbf{r}_{\mathrm{C}}, \mathbf{r}_{\mathrm{O}}, \mathbf{r}_{i}\right)+V_{\mathrm{co}}\left(\left|\mathbf{r}_{\mathrm{C}}-\mathbf{r}_{\mathrm{O}}\right|\right),
$$

where $\mathbf{r}_{c}$ and $\mathbf{r}_{o}$ are, respectively, the carbon and the oxygen position vectors and $\mathbf{r}_{i}$ are those of the $i$ th coordinate of the copper atom. The intramolecular term $V_{\text {co }}$ is a standard Morse potential

$$
\begin{aligned}
V_{\mathrm{CO}}\left(\left|\mathbf{r}_{\mathrm{C}}-\mathbf{r}_{\mathrm{O}}\right|\right)= & F\left\{\exp \left(-2 \gamma\left(\left|\mathbf{r}_{\mathrm{C}}-\mathbf{r}_{\mathrm{O}}\right|-r_{\mathrm{Co}}\right)\right)\right. \\
& \left.-2 \exp \left(-\gamma\left(\left|\mathbf{r}_{\mathrm{C}}-\mathbf{r}_{\mathrm{O}}\right|-r_{\mathrm{Co}}\right)\right)\right\},
\end{aligned}
$$

where $r_{\mathrm{co}}$ is the equilibrium distance, and the interaction of $\mathrm{CO}$ with each copper atom is described by the following modified Morse $\mathrm{C}-\mathrm{Cu}$ interaction potential:

$$
\begin{aligned}
V_{i}\left(\mathbf{r}_{\mathrm{c}}, \mathbf{r}_{\mathrm{O}}, \mathbf{r}_{i}\right)= & A \exp \left(-\alpha\left|\mathbf{r}_{i}-\mathbf{r}_{\mathrm{O}}\right|\right) \\
& +B \exp \left(-2 \beta\left(\left|\mathbf{r}_{i}-\mathbf{r}_{\mathrm{c}}\right|-r_{\mathrm{eq}}\right)\right) \\
& -2 B \cos \phi^{2} \exp \left(-\beta\left(\left|\mathbf{r}_{i}-\mathbf{r}_{\mathrm{C}}\right|-r_{\mathrm{eq}}\right)\right),
\end{aligned}
$$

where $r_{\mathrm{eq}}$ is a given equilibrium distance and

$$
\cos ^{2} \phi=\frac{\left(\mathbf{r}_{i}-\mathbf{r}_{\mathrm{C}}\right) \cdot\left(\mathbf{r}_{\mathrm{C}}-\mathbf{r}_{\mathrm{O}}\right)}{\left|\mathbf{r}_{i}-\mathbf{r}_{\mathrm{C}}\right|\left|\mathbf{r}_{\mathrm{C}}-\mathbf{r}_{\mathrm{O}}\right|}
$$

The first term in Eq. (29) describes the oxygen-copper repulsion. The remainder of the terms in Eq. (29) represent the carbon-copper interaction. The molecule orientation is taken into account by the angle $\phi$ between the $\mathrm{C}-\mathrm{O}$ and the $\mathrm{Cu}-\mathrm{C}$ bonds. The metal is represented by three layers of $36(6 \times 6)$ copper atoms arranged according to a $f c c$ lattice structure. In this work, the molecular axis is fixed perpendicular to the copper surface and the resulting bidimensional molecular motion describes the internal CO mode at $2084 \mathrm{~cm}^{-1}$ and the stretching mode perpendicular to the surface at $353 \mathrm{~cm}^{-1}$. Potential parameters are reported in Ref. 37. In this system there is no degeneracy induced by symmetry considerations. However, given the small value of the external stretch mode, it can happen that overtones of different quanta are located very close in energy space.

The De Leon-Heller method described in Sec. II is applied to the calculation of the overlap according to Eq. (20). For each eigenfunction a classical trajectory is chosen with initial conditions given by the positions at the equilibrium geometry and momenta such that $\sum_{i} p_{i}^{2} / 2 m=E$, where the sum is over each dimension. Whether the set of values $E$ of the eigenvalues, used to calculate the initial momenta and the phase of Eq. (10), were calculated using a SC-IVR with a single trajectory or MC-SC-IVR, is indicated by the subscripts. For $E_{D V R}$ the "exact" eigenvalues' set was chosen, while for $E_{1 \text { traj }}$ the single trajectory (without separable approximation) spectra values were chosen. ${ }^{34}$ In Table I one can also find the values of the frequencies evaluated for each trajectory using Eq. (11). These are comparable with the harmonic values and they do not change significantly by changing the trajectories' initial velocities. If the potential had been harmonic, the prefactor would indeed be the complex exponentiation of the mode frequency over $\hbar$. Finally, in Table I we report an index labeled as "sum" which is indicative of the overall performance of the method. A closer look at Table I shows how the frequencies are the same within less than $1 \%$, using both the set $E_{1 t r a j}$ and $E_{D V R}$ as initial conditions. Instead, significant differences can be observed for the overlap values. Clearly, the exact set of energies gives more accurate results. This shows that the De Leon-Heller method can be considered accurate enough for most eigenfunction calculation purposes, when accurate eigenvalues are known.

In Table II single-trajectory results are reported, using Eq. (17). In the second column, the trajectory used for the eigenfunction calculations for all states is the same, i.e., the ground state harmonic trajectory. It is evident that this 
TABLE I. Overlaps $O$ between exact DVR eigenfunctions and De LeonHeller eigenfunctions for $\mathrm{CO}$ on $\mathrm{Cu}(100)$ analytic potential. In the first column the quantum state is labeled by the quantum numbers of the internal and external mode, respectively. In the second and fourth columns the values of the prefactor frequencies calculated with Eq. (11). In the third column the overlap calculated using the eigenvalues $E_{n}$ obtained from the power spectrum of a single classical trajectory (Refs. 34 and 35), while in the last column using the DVR eigenvalues. The last row contains the sum of the overlaps in the columns.

\begin{tabular}{ccccc}
\hline \hline States & $\omega_{1}, \omega_{2}$ & $O\left(E_{1 t r a j}\right)$ & $\omega_{1}, \omega_{2}$ & $O\left(E_{D V R}\right)$ \\
\hline ZEP & $2061.8,335.6$ & 0.99890 & $2061.8,335.6$ & 0.99921 \\
$(0,1)$ & $2069.1,324.6$ & 0.98565 & $2064.2,319.2$ & 0.98565 \\
$(0,2)$ & $2066.3,304.5$ & 0.29482 & $2067.3,306.3$ & 0.97162 \\
$(1,0)$ & $2046.3,341.1$ & 0.99764 & $2046.7,341.1$ & 0.99073 \\
$(1,1)$ & $2044.6,322.8$ & 0.97876 & $2044.8,322.9$ & 0.97976 \\
$(1,2)$ & $2039.8,302.2$ & 0.96892 & $2031.2,282.1$ & 0.97596 \\
$(2,0)$ & $2017.2,320.9$ & 0.99855 & $2015.0,314.8$ & 0.99022 \\
$(2,1)$ & $2016.8,359.4$ & 0.97529 & $2015.7,319.9$ & 0.98323 \\
$(2,2)$ & $2018.0,299.0$ & 0.85167 & $2019.1,300.7$ & 0.96297 \\
Sum & & 8.05020 & & 8.83935 \\
\hline \hline
\end{tabular}

crude approach gradually fails as higher vibrational levels are reached, and already is quite inaccurate for the $(2,2)$ state where the overlap is $90 \%$ too small. This shows how the error increases if one attempts to use a unique trajectory to calculate states which are further apart from the energy shell of the single trajectory (see Appendix A of Ref. 59). However, this trajectory shows to be quite accurate for the first vibrational eigenstates. In the following two columns, the overlaps for trajectories with harmonic and anharmonically corrected initial momenta at the energy eigenvalues are reported. For all these columns as for the one labeled "ground," the energy eigenvalues were taken from the single trajectory power spectrum. ${ }^{34}$ From the second to the fourth column, the accuracy gradually increases, as indicated by the index "sum." Nevertheless, for the $(2,2)$ eigenstates the missed part of the overlap decreases from $57 \%$ to $40 \%$ when using the anharmonic correction. This result is still not satisfying. In the last

TABLE II. Single trajectory SC-IVR methods' eigenfunctions' overlaps for $\mathrm{CO}$ on $\mathrm{Cu}(100)$ potential. In the first column, the same notations as in Table I. In the following three columns Eq. (17) is employed using, respectively, a single ground harmonic trajectory for all eigenfunctions, eigen-trajectories with harmonic and anharmonic corrected initial conditions, respectively. The last column is the same as the previous one but using the approximation of Eq. (16) and the energy levels $E_{n}$ from the power spectrum calculated with the separable approximation (Refs. 34 and 35).

\begin{tabular}{ccccc}
\hline \hline States & $\begin{array}{c}O\left(E_{1 \text { traj }}\right) \\
\text { Ground }\end{array}$ & $\begin{array}{c}O\left(E_{1 \text { traj }}\right) \\
\text { Harmonic }\end{array}$ & $\begin{array}{c}O\left(E_{1 \text { traj }}\right) \\
\text { Anharm }\end{array}$ & $\begin{array}{c}O\left(E_{1 \text { traj-SEP }}\right) \\
\text { Anharm }\end{array}$ \\
\hline ZEP & 0.99566 & 0.99542 & 0.99550 & 0.99828 \\
$(0,1)$ & 0.96518 & 0.99520 & 0.99528 & 0.99420 \\
$(0,2)$ & 0.91580 & 0.97711 & 0.97885 & 0.84022 \\
$(1,0)$ & 0.98305 & 0.98359 & 0.98387 & 0.99358 \\
$(1,1)$ & 0.96189 & 0.98847 & 0.98865 & 0.99664 \\
$(1,2)$ & 0.84182 & 0.94239 & 0.94004 & 0.80430 \\
$(2,0)$ & 0.97670 & 0.96451 & 0.96570 & 0.98491 \\
$(2,1)$ & 0.90516 & 0.93615 & 0.93480 & 0.85252 \\
$(2,2)$ & 0.10385 & 0.43739 & 0.60409 & 0.39081 \\
Sum & 7.64911 & 8.22023 & 8.38678 & 7.85546 \\
\hline \hline
\end{tabular}

TABLE III. The same as in Table II but for MC-SC-IVR eigenfunctions using a total of only six trajectories. In column 4 a 4000 trajectory SC-IVR calculation is reported. The energy values are indicated as $E_{M C-S E P}$ when the MC-SC-IVR with separable approximation eigenvalues are employed, $E_{M C}$ when the ones without the separable approximation are used and $E_{D V R}$ when the DVR ones are used.

\begin{tabular}{cccccc}
\hline \hline & 6 traj.s-SEP & 6 traj.s & 4000 traj.s & 6 traj.s-SEP & 6 traj.s \\
States & $O\left(E_{M C-S E P}\right)$ & $O\left(E_{M C}\right)$ & $O\left(E_{M C}\right)$ & $O\left(E_{D V R}\right)$ & $O\left(E_{D V R}\right)$ \\
\hline ZEP & 0.99382 & 0.99485 & 0.99466 & 0.99371 & 0.98885 \\
$(0,1)$ & 0.99703 & 0.99335 & 0.99025 & 0.99329 & 0.97528 \\
$(0,2)$ & 0.94993 & 0.93663 & 0.98930 & 0.97879 & 0.96466 \\
$(1,0)$ & 0.99423 & 0.81562 & 0.92798 & 0.95208 & 0.80860 \\
$(1,1)$ & 0.97920 & 0.99255 & 0.95964 & 0.99397 & 0.99197 \\
$(1,2)$ & 0.99344 & 0.98446 & 0.97919 & 0.99086 & 0.98850 \\
$(2,0)$ & 0.97475 & 0.98746 & 0.98009 & 0.98401 & 0.98760 \\
$(2,1)$ & 0.98489 & 0.98467 & 0.95010 & 0.97326 & 0.96496 \\
$(2,2)$ & 0.98685 & 0.97912 & 0.91446 & 0.92457 & 0.98088 \\
Sum & 8.85414 & 8.66871 & 8.68567 & 8.78460 & 8.65130 \\
\hline \hline
\end{tabular}

column, the same calculations as in the fourth column are performed, but using a consistent (i.e., with the eigenvalue at the same semiclassical level of calculation) set of separable approximation results for the power spectra and eigenfunction evaluation according to Eq. (16). The separable approximation clearly generate poorer results than the ones reported on the fourth column, where this approximation was not invoked.

Finally Eqs. (18) and (16) are employed for eigenfunction calculations. The results are reported in Table III for different sets of trajectories and for different levels of approximation. These calculations are evidence of the substantial increase of accuracy due to the use of multiple trajectories. In the second column, we report the results of using the MC-SCIVR approach, where the separable approximation was used both for the eigenfunctions and power spectrum calculations, in order to have a set of consistent results. These results are accurate for our purposes, as shown by the "sum" index. In particular the higher vibrational state taken in this set of calculation, i.e., the $(2,2)$ state, is exact within $1 \%$ of accuracy. Exactly the same considerations are valid for a second set of MC-SC-IVR calculations reported on the following column. Here the separable approximation is not employed and the accuracy of the results is slightly less. In the column labeled "4000 traj.s," the fully converged semiclassical limit is reached using a 4000-classical trajectory calculation for Eq. (15) with 4000 time steps of 10 a.u. each. Here the eigenvalues coming from MC-SC-IVR calculations ${ }^{35}$ are used, i.e., the same ones as in the previous column. Thus, the comparison between these two columns shows the accuracy of our MC-SC-IVR method for eigenfunction calculations respect to the original HHKK semiclassical formulation. Differences between the two sets of overlaps are very small and the MC-SC-IVR method, both with or without the separable approximation, can be used with only few trajectories (six in this case) as a good estimate of the actual semiclassical calculation for vibrational eigenfunctions. These results confirm the previous power spectra calculation ones, ${ }^{35}$ showing that the MC-SC-IVR can properly mimic quantum properties for this kind of bound states at the fully converged SC-IVR level of calculation, with much less effort. In the 


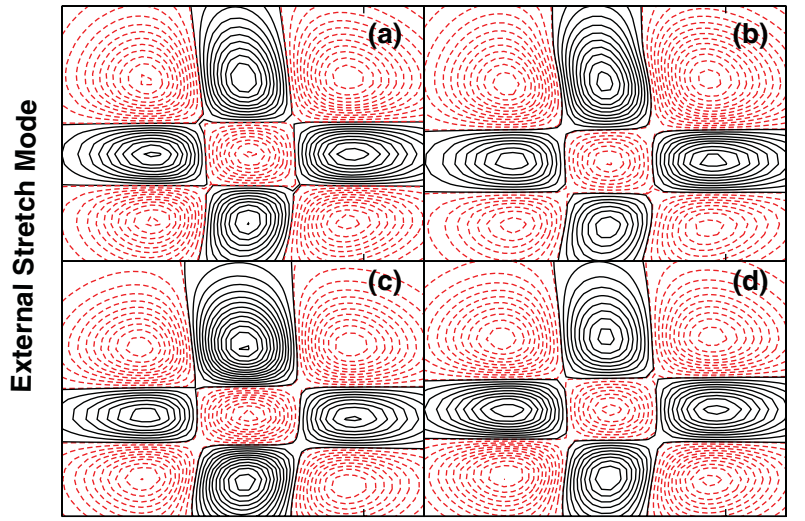

Internal Stretch Mode

FIG. 2. Normalized vibrational eigenfunction contour plots for the $(2,2)$ eigenstate at the exact DVR energy for different methods: (a) the exact DVR eigenfunction; (b) using De Leon-Heller method; (c) from MC-SC-IVR with the approximation of Eq. (16); (d) without invoking the approximation in Eq. (16). Dashed red lines are for negative values.

last two columns of Table III the same calculations as in the second and third columns are, respectively, reported, but using the exact eigenvalues $E_{n}$ in Eq. (18). Once again, one can observe that the overlaps are not significantly different from those in the second and third columns and they are all very accurate, up to the $(2,2)$ vibrational state.

A graphical comparison is even more striking than the numerical one presented in the previous tables. In Fig. 1 the eigenfunction methods used for calculating the overlaps reported previously are depicted. On panel (a)-(f) the $(2,2)$ eigenfunctions are represented on contour plots, where the dashed color lines are the negative contour of the eigenfunctions. Panel (a) is the exact DVR eigenfunction. Panel (b) eigenfunction is calculated using the ground state trajectory and one can observe how it completely misses the correct nodal arrangement. On panel (c) the De Leon-Heller method wave function is plotted using a single trajectory eigenvalue, while on panel (d) Eq. (17) is used with the same set of eigenvalues as for the previous panel. Finally on panels (e) and (f) the MC-SC-IVR eigenfunctions are reported, respectively, with and without the separable approximation. These last plots of eigenfunctions are clearly more accurate

TABLE IV. Values of $\varepsilon_{1}$ of Eq. (21) for different semiclassical methods. The second row indicates the semiclassical method used for calculating the energy in the Fourier transform, as described in previous tables.

\begin{tabular}{ccccc}
\hline \hline & 1 traj.-SEP & 1 traj. & 6 traj.s-SEP & 6 traj.s \\
States & $O\left(E_{1 \text { traj-SEP }}\right)$ & $O\left(E_{1 \text { traj }}\right)$ & $O\left(E_{M C-S E P}\right)$ & $O\left(E_{M C}\right)$ \\
\hline ZEP & $1.104 \times 10^{-1}$ & $1.152 \times 10^{-1}$ & $9.8576 \times 10^{-2}$ & $1.2162 \times 10^{-1}$ \\
$(0,1)$ & $8.784 \times 10^{-2}$ & $9.095 \times 10^{-2}$ & $6.7365 \times 10^{-2}$ & $9.3723 \times 10^{-2}$ \\
$(0,2)$ & $8.028 \times 10^{-2}$ & $8.668 \times 10^{-2}$ & $1.2418 \times 10^{-1}$ & $2.4030 \times 10^{-1}$ \\
$(1,0)$ & $7.240 \times 10^{-2}$ & $7.966 \times 10^{-2}$ & $4.4660 \times 10^{-2}$ & $1.9413 \times 10^{-1}$ \\
$(1,1)$ & $3.018 \times 10^{-2}$ & $5.722 \times 10^{-2}$ & $4.0150 \times 10^{-2}$ & $4.7557 \times 10^{-2}$ \\
$(1,2)$ & $8.621 \times 10^{-2}$ & $9.182 \times 10^{-2}$ & $2.4551 \times 10^{-2}$ & $6.6439 \times 10^{-2}$ \\
$(2,0)$ & $7.291 \times 10^{-2}$ & $7.334 \times 10^{-2}$ & $5.2488 \times 10^{-2}$ & $5.0164 \times 10^{-2}$ \\
$(2,1)$ & $6.986 \times 10^{-2}$ & $7.080 \times 10^{-2}$ & $4.5017 \times 10^{-2}$ & $5.2962 \times 10^{-2}$ \\
$(2,2)$ & $8.935 \times 10^{-2}$ & $9.281 \times 10^{-2}$ & $4.0819 \times 10^{-2}$ & $5.9060 \times 10^{-2}$ \\
Sum & $69.94 \times 10^{-2}$ & $75.85 \times 10^{-2}$ & $42.60 \times 10^{-2}$ & $53.50 \times 10^{-2}$ \\
\hline \hline
\end{tabular}

TABLE V. The same as in Table IV but for $\varepsilon_{2}$.

\begin{tabular}{ccccc}
\hline \hline States & $\begin{array}{c}1 \text { traj.SEP } \\
O\left(E_{1 \text { traj-SEP }}\right)\end{array}$ & $\begin{array}{c}1 \text { traj. } \\
O\left(E_{1 \text { traj }}\right)\end{array}$ & $\begin{array}{c}6 \text { traj.s-SEP } \\
O\left(E_{M C-S E P}\right)\end{array}$ & $\begin{array}{c}6 \text { traj.s } \\
O\left(E_{M C}\right)\end{array}$ \\
\hline ZEP & 1.0141 & 1.0153 & 1.0127 & 1.0171 \\
$(0,1)$ & 1.0112 & 1.0121 & 1.0070 & 1.0132 \\
$(0,2)$ & 1.0144 & 1.0145 & 1.0291 & 1.1083 \\
$(1,0)$ & 1.0160 & 1.0193 & 1.0061 & 1.1272 \\
$(1,1)$ & 1.0022 & 1.0104 & 1.0057 & 1.0064 \\
$(1,2)$ & 1.0270 & 1.0309 & 1.0014 & 1.0156 \\
$(2,0)$ & 1.0243 & 1.0248 & 0.99244 & 1.0073 \\
$(2,1)$ & 1.0223 & 1.0237 & 0.99563 & 1.0086 \\
$(2,2)$ & 1.0460 & 1.0492 & 0.99810 & 1.0115 \\
\hline \hline
\end{tabular}

than other ones, showing that the MC-SC-IVR method offers a consistent formulation for eigenvalue and eigenfunction calculations.

In order to find the origin of the consistent accuracy of the methods on panel (b) to (c), on Fig. 2, the eigenfunctions of the MC-SC-IVR and De Leon-Heller semiclassical methods at the exact $(2,2)$ vibrational energy level are reported, together with the exact one represented on panel (a). This time all semiclassical methods faithfully reproduce the nodal behavior of the exact eigenfunction. This clearly shows that the performance of the method depends mostly on the accuracy of the calculation of the eigenvalues and that MC-SC-IVR in Eq. (18) and De Leon-Heller method in Eqs. (9)-(11) are almost numerically equivalent.

Now, let us turn to the estimate of the eigenfunctions' accuracy via the coefficients $\varepsilon_{1}$ and $\varepsilon_{2}$ presented in Sec. III. Even if the $\mathrm{CO}$ on $\mathrm{Cu}(100)$ vibrational system is not degenerate, we can understand how accurate these parameters are by comparing their values in this non-degenerate case to other degenerate cases. In Table IV, the values of $\varepsilon_{1}$ are calculated for each vibrational state. Since $\varepsilon_{1}$ is the sum of the absolute value of the deviation of the approximate method from the exact one at each DVR point, there are no error compensations and it represents an upper bound for the error estimate. On the second and third columns the single trajectory results are reported, while on the following columns the multiple trajectory results are presented. Once again the indicative index "sum" shows that overall the multiple-trajectories perform best, with an improvement of accuracy of almost $50 \%$. This means that the MC-SC-IVR eigenfunctions mimic the DVR ones better than the single trajectory calculations. However, given such small values of $\varepsilon_{1}$ for all methods, one can consider the sin-

TABLE VI. Overlap of the ground state vibrational $\mathrm{CO}_{2}$ eigenfunctions at different semiclassical levels of calculation with the exact DVR eigenfunctions.

\begin{tabular}{lc}
\hline \hline Method & Overlap \\
\hline 1 traj.-SEP & 0.99953 \\
1traj & 0.99893 \\
De Leon-Heller & 0.99663 \\
MCSC-IVR-sep & $0.99953^{\mathrm{a}}$ \\
MCSC-IVR & $0.99669^{\mathrm{a}}$ \\
\hline
\end{tabular}

${ }^{\mathrm{a}}$ The first four eigenstates trajectories have been used. 

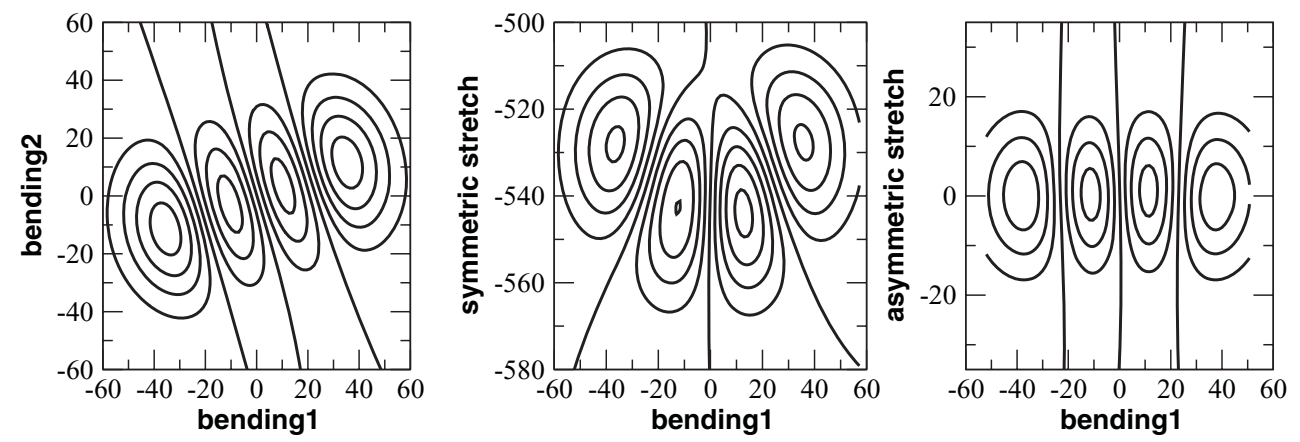

FIG. 3. Bidimensional vibrational eigenfunction contour plot cuts for the state $\left(0,3^{1}, 0\right)$ of $\mathrm{CO}_{2}$, i.e., the triple bending excitation. The nodes are significantly tilted in the bending and symmetric stretch subspaces, while asymmetric stretch has a small coupling with the bending stretches subspace.

gle trajectory results to be quite accurate as well. A close comparison of the multiple trajectory results reveals that the MC-SC-IVR-SEP is slightly more accurate as found before in the overlap calculation. However, this improvement is really small and not significant.

In Table $\mathrm{V}$ the values of the coefficient $\varepsilon_{2}$ are given for each vibrational state. Since the $\mathrm{CO}$ on $\mathrm{Cu}(100)$ does not present degeneracy, ${ }^{61}$ in this case $\varepsilon_{2}$ is an estimate of the $i$ th semiclassical eigenfunction's orthogonality to all the DVR eigenfunctions except the $i$ th one. In other words, these calculations measure if there is significant overlap between the $i$-esime semiclassical eigenfuction and the DVR eigenfunctions of all other quantum states. Any deviation from unit quantifies the amount of overlap. As one can see from Table V, these deviations are really small and this shows from a different perspective the results of the previous tables. All methods are accurate within two digits and the multipletrajectory ones are still more accurate. The critical eigenstate is the $(2,2)$ state where a distinction can be made between the single and the multiple trajectory methods. Since in this case there is no degeneracy, the values of $\varepsilon_{2}$ in Table $\mathrm{V}$ were not necessary. However, these value will represent a term of comparison for degenerate cases of Sec. IV where the overlaps of Eq. (20) are not always calculated.

\section{FIRST-PRINCIPLES CALCULATION OF VIBRATIONAL EIGENFUNCTIONS}

The full dimensional on-the-fly calculation of the vibrational eigenfunctions of the $\mathrm{CO}_{2}$ molecule is a challenging test for our semiclassical methods. The four vibrational modes are strongly coupled and numerous Fermi resonances originate from the inter-play between the symmetric stretch and the bending modes. In these cases, there is a specific link between classical trajectories and the corresponding quantum phenomenology, as shown by Heller ${ }^{21}$ in model resonating systems. In other words, there is a quantum to classical correspondence that directly parallels the strong resonance effects observed classically to the quantum properties. This suggests that semiclassical methods are able to reproduce resonating quantum features.

Previous on-the-fly calculations of the vibrational eigenvalues have proved to be very accurate if compared to exact grid methods, even when few trajectories were employed. ${ }^{34,35}$ In this section vibrational eigenfunctions will also be calculated using Born-Oppenheimer classical molecular trajectories, where all the semiclassical propagator components are calculated directly from the electronic structure at the level of DFT. For example, the Hessian matrix which is required at each time step for the prefactor calculation of Eq. (14), is calculated from the second derivative operator in the Kohn and Sham formalism. In this way, the vibrational eigenfunction depicted in Fig. 3 has been calculated.

This eigenfunction corresponds to a triple-bending excited state. The distortion of nodal planes and caustic envelopes (the outer edge of the wave functions) are clear signs of non-linear strong couplings. The nodal line shapes are equally tilted in the bending subspace, which reveals the degeneracy between these two modes. Instead, in the bending and symmetric stretch subspace, the three nodes are arranged
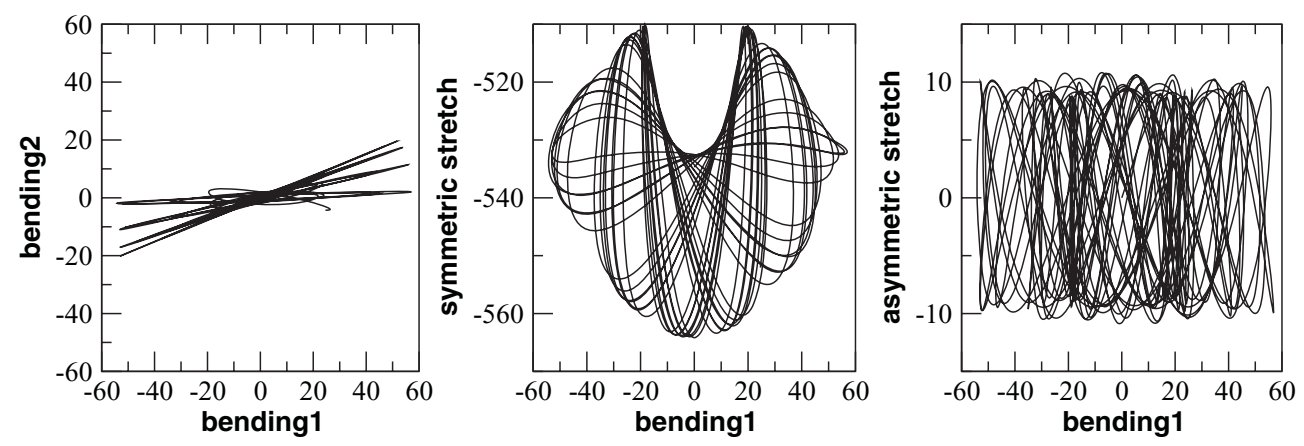

FIG. 4. Bidimensional plots for the "eigen-trajectory" with initial conditions associated to the $\left(0,3^{1}, 0\right)$ vibrational state of $\mathrm{CO}_{2}$. The vibrational eigenfunctions of Fig. 3 are visibly on correspondence with this classical trajectory. 
TABLE VII. Values of $\varepsilon_{1}$ of Eq. (21) for different semiclassical methods. On the first row the name of the semiclassical method is reported.

\begin{tabular}{|c|c|c|c|c|c|}
\hline $\begin{array}{c}E_{D V R} \\
{\left[\mathrm{~cm}^{-1}\right]}\end{array}$ & 1 traj.-SEP & 1 traj. & De Leon-Heller & MCSC-IVR-sep & MCSC-IVR \\
\hline 2504.6 & $7.77 \times 10^{-4}$ & $1.09 \times 10^{-3}$ & $1.28 \times 10^{-3}$ & $9.63 \times 10^{-4}$ & $1.46 \times 10^{-3 \mathrm{a}}$ \\
\hline 3143.8 & $1.05 \times 10^{-3}$ & $1.06 \times 10^{-3}$ & $5.45 \times 10^{-4}$ & $1.70 \times 10^{-3}$ & $2.59 \times 10^{-3}$ \\
\hline 3752.0 & $1.73 \times 10^{-3}$ & $9.72 \times 10^{-4}$ & $8.15 \times 10^{-4}$ & $6.75 \times 10^{-4}$ & $2.42 \times 10^{-3}$ \\
\hline 3871.4 & $7.90 \times 10^{-4}$ & $1.97 \times 10^{-3}$ & $1.88 \times 10^{-3}$ & $8.15 \times 10^{-4}$ & $5.67 \times 10^{-3}$ \\
\hline Sum & $4.35 \times 10^{-3}$ & $5.09 \times 10^{-3}$ & $4.52 \times 10^{-3}$ & $4.15 \times 10^{-3}$ & $12.14 \times 10^{-3}$ \\
\hline
\end{tabular}

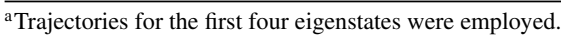

in order to be perpendicular to a $\mathrm{U}$ shaped line connecting the maximum of the eigenfunction. In the bending versus asymmetric stretch subspace the nodal lines are straight and perpendicular to the bending mode showing that no significant coupling is present between the two modes.

As anticipated above, this inspection of nodal patterns and their distortion can be explained in terms of classical trajectories. ${ }^{59}$

By looking at the first panel on the left of Fig. 4, one can see how this periodic trajectory is closing onto itself, an evidence of the synchronization between the two bending modes. An opposite behavior is reported on the last panel where the subspace is almost entirely filled. More interesting is the central panel where the hallmark of the Fermi resonance is given by the $\mathrm{U}$ shape resulting from the convolution of the classical trajectory. By comparing the central panels of Figs. 3 and 4 , one can see how the vibrational eigenfunction corresponds to that of the classical trajectory convolution. Further, quantum delocalization effects are indeed taken into account in our semiclassical approximation. In fact, let us compare the right panels of these figures: in Fig. 4 the classical trajectories range from -10 to +10 (in normal modes mass scaled units) along the asymmetric subspace, while in Fig. 3 the eigenfunction is delocalized to occupy almost double the interval.

In order to evaluate the accuracy of the semiclassical eigenfunctions, a set of DVR calculations were performed. In particular, the Hamiltonian operator was diagonalized in coordinate space using a sparse matrix representation interfaced with the ARPACK library diagonalization routines. ${ }^{60}$ Only the first 20 eigenvalues and eigenfunctions of the matrix were computed to reduce the computational memory request. To obtain higher eigenvalues more grid points are needed and hence more memory space is requested to store the matrix to be diagonalized.

The Hamiltonian operator is expressed in the sinc-DVR formalism in four dimensions (one for each mode) ${ }^{55}$ The potential energy surface employed is that of the analytical potential obtained by fitting the data on the fly. ${ }^{34}$ After various tests the converged set of eigenvalues and eigenfunctions were obtained with 52000 points grid. A ten times denser grid provided the same eigenvalues up to the fourth significant figure and the error for the exact eigenfunctions (i.e., the value of parameter $\varepsilon_{1}$ ) is of the order of machine precision. In the diagonalization routine employed, the accuracy of the eigenvectors was checked to be less than $10^{-12}$ for the given eigenvalues, i.e., once the eigenvalues have converged up to the fourth decimal place the eigenfunctions corresponding to such eigenvalues are correct to within machine precision.

Such exact eigenfunctions were used to determine the accuracy of the semiclassical ones. The direct overlap comparison was possible only for the ground state, because most of the higher states are degenerate.

In Table VI the overlaps for different levels of semiclassical calculations are reported. One can conclude that all methods are accurate.

In order to have a complete picture of how all methods perform for the first few vibrational states calculated by DVR, a set of calculations for $\varepsilon_{1}$ and $\varepsilon_{2}$ are reported in Tables VII and VIII.

On the first row the different semiclassical methods are labeled and on the first column the exact eigenvalues of the vibrational levels are given. We remember that an exact eigenfunction will have a value of $\varepsilon_{1}=0$ and the values of $\varepsilon_{1}$ reported in Table VII are all of the order of hundredths.
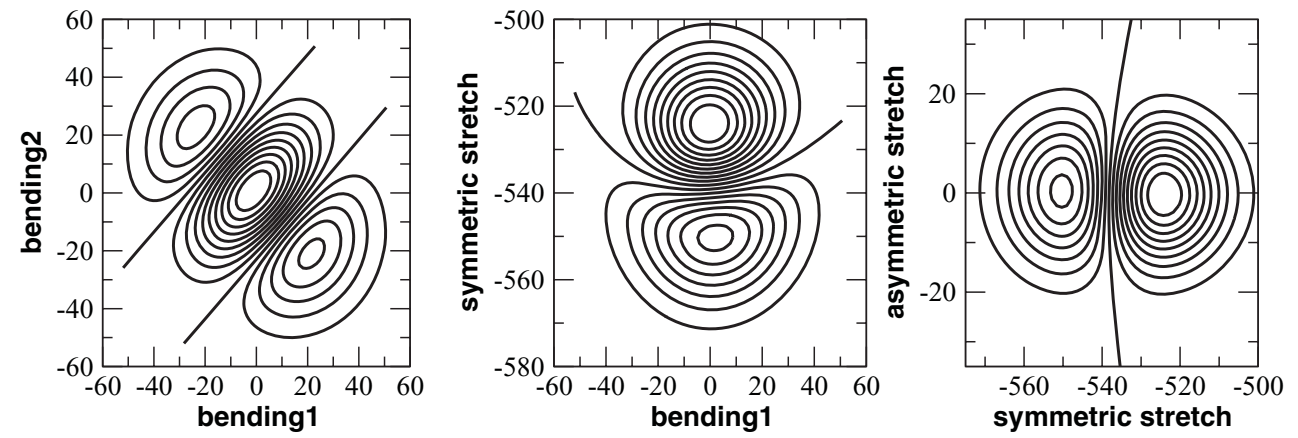

FIG. 5. Bidimensional vibrational eigenfunction contour plot cuts for the $\mathrm{CO}_{2}$ vibrational state $\left(1,0^{0}, 0\right)$, i.e., the single symmetric stretch excitation. The nodal line in the bending/symmetric subspace is U shaped, in correspondence with the 2:1 Fermi resonance. The asymmetric stretch has a small coupling with the bending stretch. 

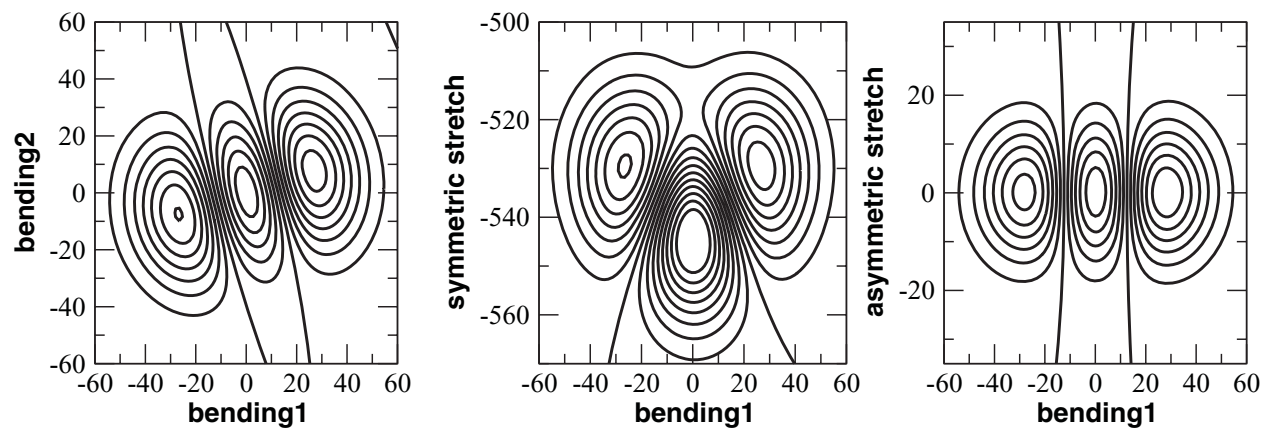

FIG. 6. Bidimensional vibrational eigenfunction contour plot cuts for the $\mathrm{CO}_{2}$ vibrational state $\left(0,2^{0}\right.$, 0$)$, i.e., the double bending excitation. As in Fig. 5 the nodal line in the bending/symmetric subspace is $\mathrm{U}$ shaped, but in a symmetric fashion. The asymmetric stretch has a small coupling with the bending stretch.

However, in order to quantify the overall accuracy of each method on the last row of Table VII the sums of the values in each column are reported. Even these sums are still of the order of hundredths and any distinction between the semiclassical methods is quite superfluous. The same considerations are valid for $\varepsilon_{2}$, where the exact value is $\varepsilon_{2}=1$.

From Table VIII, we note that all semiclassical methods are very accurate. Instead, when calculating the overlap of a single degenerate semiclassical eigenfunction with the DVR eigenfunction we found errors even of the order of $50 \%$. As discussed previously, the introduction of the coefficients $\varepsilon_{1}$ and $\varepsilon_{2}$ is indeed necessary to evaluate accuracy of the degenerate eigenfunctions.

The most interesting vibrational states are the Fermi resonating states. The states labeled by a single symmetric stretch excitation and a double bending are represented, respectively, in Figs. 5 and 6.

Since twice the bending frequency is about a single symmetric stretch excitation, these states resonate and split into the Fermi states reported in Figs. 5 and 6. In these plots, the bending subspaces are as usual synchronized as shown by the tilted nodal lines. Instead, in the symmetric/asymmetric (Fig. 5) and in the bending/asymmetric (Fig. 6) subspace the nodal lines are perpendicular to the excited mode, showing that no significant coupling is present between the asymmetric stretch mode and the other ones. More interesting is the bending/symmetric stretch subspace in both figures, where the nodal line is U-shaped. In Fig. 5 the $\mathrm{U}$ shaped nodal line is pointing upwards, while it points downwards in Fig. 6. This shape is typical of a 2:1 Fermi resonance and one can also detect it from the envelope of the corresponding classical trajectories' subspace.

TABLE VIII. Values of $\varepsilon_{2}$ of Eq. (22) for different semiclassical methods. On the first row the name of the semiclassical method is reported for the same simulations as in Table VII.

\begin{tabular}{cccccc}
\hline \hline $\begin{array}{c}E_{D V R} \\
{\left[\mathrm{~cm}^{-1}\right]}\end{array}$ & 1 traj.SEP & 1 traj. & $\begin{array}{c}\text { De Leon- } \\
\text { Heller }\end{array}$ & $\begin{array}{c}\text { MCSC- } \\
\text { IVR-sep }\end{array}$ & $\begin{array}{c}\text { MCSC- } \\
\text { IVR }\end{array}$ \\
\hline 2504.6 & 1.0006 & 1.0012 & 1.0025 & 1.0012 & $1.0033^{\mathrm{a}}$ \\
3143.8 & 1.0018 & 1.0020 & 1.0007 & 1.0043 & 1.0114 \\
3752.0 & 1.0084 & 1.0045 & 1.0033 & 1.0024 & 1.0183 \\
3871.4 & 0.9991 & 1.0016 & 0.9932 & 0.9992 & 1.0602 \\
\hline
\end{tabular}

${ }^{a}$ Trajectories for the first four eigenstates were employed.

\section{CONCLUSIONS}

In this paper a semiclassical initial value representation method (MC-SC-IVR) has been implemented for the calculation of vibrational eigenfunctions. It has been compared with other semiclassical methods and they all result equally good. The fact that these methods, implemented using an on-the-fly approach, are accurate enough to correctly describe a complex quantum system such as the $\mathrm{CO}_{2}$ molecule is noteworthy. In conclusion, we have observed that none of the methods employed can be considered superior to the others and that the accuracy of the semiclassical eigenfunction is mainly dictated by the accuracy of the corresponding eigenvalue calculation. To explore the role of the choice of trial eigenvalue, we scanned the eigenvalue of Eq. (8) and observed how the overlap of Eq. (20) changed as a function of the scanned eigenvalue. The numerical results show that a resolution in the spectrum better than $10 \mathrm{~cm}^{-1}$ is required to have a few percent of eigenfunction overlap error. Finally, these eigenfunctions can be used as guiding functions for more accurate Monte Carlo calculations. Work in this direction is in progress in our groups.

\section{ACKNOWLEDGMENTS}

The University of Milan is thanked for funding (PUR grant) and CILEA (Consorzio Interuniversitario Lombardo per L'Elaborazione Automatica) for computational time allocation. Authors also thank FAS Research Computing for computational support. S.V. acknowledges support from the National Science Foundation (NSF) SOLAR project (Grant No. DMR-0934480). A.A.-G. acknowledges support from the Sloan Foundation and the Camille and Henry Dreyfus foundation.

${ }^{1}$ X.-G. Wang and T. Carrington, Jr., J. Chem. Phys. 130, 094101 (2009).

${ }^{2}$ R. B. Lehoucq, S. K. Gray, D.-H. Zhang, and J. C. Light, Comput. Phys. Commun. 109(1), 15 (1998).

${ }^{3}$ J. Echave and D. C. Clary, Chem. Phys. Lett. 190, 225 (1992).

${ }^{4}$ J. Tennyson and J. R. Henderson, J. Chem. Phys. 91, 3815 (1989); B. T. Sutcliffe and J. Tennyson, Int. J. Quantum Chem. 29, 183 (1991); 42, 941 (1992).

${ }^{5}$ B. Q. Li, C. Mollica, and J. Vanicek, J. Chem. Phys. 131, 041101 (2009).

${ }^{6}$ T. Zimmermann and J. Vanicek, J. Chem. Phys. 132, 241101 (2010).

${ }^{7}$ T. Zimmermann, J. Ruppen, B. Q. Li, and J. Vanicek, Int. J. Quantum Chem. 110, 2426 (2010).

${ }^{8}$ J. M. Herbert and M. Head-Gordon, Phys. Chem. Chem. Phys. 7, 3269 (2005). 
${ }^{9}$ R. Car and M. Parrinello, Phys. Rev. Lett. 55, 2471 (1985).

${ }^{10}$ H. B. Schlegel, J. M. Millam, S. S. Iyengar, G. A. Voth, A. D. Daniels, G. E. Scuseria, and M. J. Frisch, J. Chem. Phys. 114, 9758 (2001).

${ }^{11}$ J. M. Herbert and M. Head-Gordon, J. Chem. Phys. 121, 11542 (2004).

${ }^{12}$ Y. Liu, D. Yarne, and M. E. Tuckerman, Phys. Rev. B 68, 125110 (2003).

${ }^{13}$ M. Pavese, D. R. Berard, and G. A. Voth, Chem. Phys. Lett. 300, 93 (1999).

${ }^{14}$ G. A. Worth, M. A. Robb, and I. Burghardt, Faraday Discuss. 127, 307 (2004).

${ }^{15}$ S. Iyengar and J. Jakowski, J. Chem. Phys. 122, 114105 (2005).

${ }^{16}$ O. Knospe and P. Jungwirth, Chem. Phys. Lett. 317, 529 (2000).

${ }^{17}$ J. Tatchen and E. Pollak, J. Chem. Phys. 130, 041103 (2009).

${ }^{18}$ A. Einstein, Dent. Ges. Berlin Verh. 19, No. 9/10 (1917); an English translation by C. Jaffe is available as Joint Institute for Laboratory and Astrophysics, Boulder, Colorado, Technical Report No. 16 (unpublished); J. B. Keller, Ann. Phys. (N.Y.) 4, 180 (1950).

${ }^{19}$ S. K. Knudson, J. B. Delos, and D. W. Noid, J. Chem. Phys. 84, 6886 (1986).

${ }^{20}$ H. Jeffreys, Proc. London Math. Soc. 23, 428 (1923); G. Wentzel, Z. Phys. 38, 518 (1926); H. A. Kramers, ibid. 39, 828 (1926); L. Brillouin, Acad. Sci., Paris, C. R. 183, 24 (1926).

${ }^{21}$ E. J. Heller, E. B. Stechel, and M. J. Davis, J. Chem. Phys. 73, 4720 (1980).

${ }^{22}$ N. De Leon and E. J. Heller, J. Chem. Phys. 81, 5957 (1984).

${ }^{23}$ J. R. Reimers and E. J. Heller, J. Chem. Phys. 83, 511 (1985).

${ }^{24}$ J. R. Reimers and E. J. Heller, J. Phys. A 19, 2559 (1986).

${ }^{25}$ J. R. Reimers and E. J. Heller, J. Phys. Chem. 92, 3225 (1988).

${ }^{26}$ K. G. Kay, Phys. Rev. A 63, 042110 (2001).

${ }^{27}$ K. G. Kay, Phys. Rev. A 65, 032101 (2002).

${ }^{28}$ K. G. Kay, Phys. Rev. A 69, 062106 (2004)

${ }^{29}$ T. Sklarz and K. G. Kay, J. Chem. Phys. 117, 5988 (2002).

${ }^{30}$ S. Zhang and E. Pollak, J. Chem. Phys. 119, 11058 (2003).

${ }^{31}$ S. Zhang and E. Pollak, Phys. Rev. Lett. 91, 190201 (2003).

${ }^{32}$ G. Hochman and K. G. Kay, Phys. Rev. Lett. A 73, 064102 (2006).

${ }^{33}$ G. Hochman and K. G. Kay, J. Phys. A: Math. Theor. 41, 385303 (2008).

${ }^{34}$ M. Ceotto, S. Atahan, S. Shim, G. F. Tantardini, and A. Aspuru-Guzik, Phys. Chem. Chem. Phys. 11, 3861 (2009).

${ }^{35}$ M. Ceotto, S. Atahan, G. F. Tantardini, and A. Aspuru-Guzik, J. Chem. Phys. 130, 234113 (2009).

${ }^{36}$ W. H. Miller, J. Chem. Phys. 53, 3578 (1970); 53, 1949 (1970); W. H. Miller, J. Phys. Chem. A 105, 2942 (2001); M. Thoss and H. Wang, Annu. Rev. Phys. Chem. 55, 299 (2004); K. G. Kay, ibid. 56, 255 (2005).

${ }^{37}$ M. Ceotto, D. dell'Angelo, and G. F. Tantardini, J. Chem. Phys. 133, 054701 (2010).

${ }^{38}$ S. Y. Y. Wong, D. M. Benoit, M. Lewerenz, A. Brown, and P.-N. Roy, J. Chem. Phys. 134, 094110 (2011).

${ }^{39}$ D. J. Tannor, Introduction to Quantum Mechanics a Time-Dependent Perspective (University Science Books, Sausalito, CA, 2007).

${ }^{40}$ J. H. Frederick and E. J. Heller, J. Chem. Phys. 87, 6592 (1987).

${ }^{41}$ M. J. Davis and E. J. Heller, J. Chem. Phys. 75, 3916 (1981).

${ }^{42}$ W. H. Miller, Adv. Chem. Phys. 25, 69 (1974).

${ }^{43}$ H. Wang, X. Sun, and W. H. Miller, J. Chem. Phys. 108, 9726 (1998); X. Sun and W. H. Miller, ibid. 110, 6635 (1999); M. Thoss, H. Wang, and
W. H. Miller, ibid. 114, 9220 (2001); T. Yamamoto, H. Wang, and W. H. Miller, ibid. 116, 7335 (2002); T. Yamamoto and W. H. Miller, ibid. 118, 2135 (2003).

${ }^{44}$ M. Topaler and N. Makri, J. Chem. Phys. 101, 7500 (1994); K. Thompsoon and N. Makri, ibid. 110, 1343 (1999); N. Makri, Annu. Rev. Phys. Chem. 50, 167 (1999); N. J. Wright and N. Makri, J. Chem. Phys. 119, 1634 (2003).

${ }^{45}$ J. Ankerhold, M. Saltzer, and E. Pollak, J. Chem. Phys. 116, 5925 (2002); S. S. Zhang and E. Pollak, ibid. 121, 3384 (2004).

${ }^{46}$ A. R. Walton and D. E. Manolopoulos, Mol. Phys. 87, 961 (1996); Chem. Phys. Lett. 244, 448 (1995); M. L. Brewer, J. S. Hulme, and D. E. Manolopoulos, J. Chem. Phys. 106, 4832 (1997).

${ }^{47}$ S. Bonella, D. Montemayor, and D. F. Coker, Proc. Natl. Acad. Sci. U.S.A. 102, 6715 (2005); S. Bonella and D. F. Coker, J. Chem. Phys. 118, 4370 (2003).

${ }^{48}$ Y. Wu, M. Herman, and V. S. Batista, J. Chem. Phys. 122, 114114 (2005); Y. Wu and V. S. Batista, ibid. 118, 6720 (2003).

${ }^{49}$ F. Grossmann, Comments At. Mol. Phys. 34, 243 (1999).

${ }^{50}$ E. J. Heller, J. Chem. Phys. 62, 1544 (1975); 75, 2923 (1981).

${ }^{51}$ M. F. Herman and E. Kluk, Chem. Phys. 91, 27 (1984); K. G. Kay, J. Chem. Phys. 100, 4377 (1994); 100, 4432 (1994).

${ }^{52}$ A. Odell, A. Delin, B. Johansson, N. Bock, M. Challacombe, and A. M. N. Niklasson, J. Chem Phys. 131, 244106 (2009).

${ }^{53}$ Y. Shao, L. Fusti-Molnar, Y. Jung, J. Kussmann, C. Ochsenfeld, S. T. Brown, A. T. B. Gilbert, L. V. Slipchenko, S. V. Levchenko, D. P. O’Neill, R. A. DiStasio, Jr., R. C. Lochan, T. Wang, G. J. O. Beran, N. A. Besley, J. M. Herbert, C. Y. Lin, T. Van Voorhis, S. H. Chien, A. Sodt, R. P. Steele, V. A. Rassolov, P. E. Maslen, P. P. Korambath, R. D. Adamson, B. Austin, J. Baker, E. F. C. Byrd, H. Daschel, R. J. Doerksen, A. Dreuw, B. D. Dunietz, A. D. Dutoi, T. R. Furlani, S. R. Gwaltney, A. Heyden, S. Hirata, C.-P. Hsu, G. Kedziora, R. Z. Khaliullin, P. Klunzinger, A. M. Lee, M. S. Lee, W.-Z. Liang, I. Lotan, N. Nair, B. Peters, E. I. Proynov, P. A. Pieniazek, Y. M. Rhee, J. Ritchie, E. Rosta, C. D. Sherrill, A. C. Simmonett, J. E. Subotnik, H. L. Woodcock III, W. Zhang, A. T. Bell, A. K. Chakraborty, D. M. Chipman, F. J. Keil, A. Warshel, W. J. Hehre, H. F. Schaefer III, J. Kong, A. I. Krylov, P. M. W. Gill, and M. Head-Gordon, Phys. Chem. Chem. Phys. 8, 3172 (2006).

${ }^{54}$ A. L. Kaledin and W. H. Miller, J. Chem. Phys. 118, 7174 (2003); 119, 3078 (2003)

${ }^{55}$ D. Colbert and W. H. Miller, J. Chem. Phys. 96, 1982 (1992).

${ }^{56}$ J. C. Tully, M. Gomez, and M. Head-Gordon, J. Vac. Sci. Technol. A 11(4), 1914 (1993).

${ }^{57}$ J. T. Kindt, J. C. Tully, M. Head-Gordon, and M. A. Gomez, J. Chem. Phys. 109, 3629 (1998).

${ }^{58}$ C. W. Muhlhausen, L. R. Williams, and J. C. Tully, J. Chem. Phys. 83, 2594 (1985).

${ }^{59}$ N. De Leon and E. J. Heller, J. Chem. Phys. 78, 4005 (1983).

${ }^{60}$ R. B. Lehoucq, D. C. Sorensen, C. Yang, ARPACK Users Guide: Solution of Large-Scale Eigenvalue Problems with Implicitly Restarted Arnoldi Methods (SIAM, Philadelphia, 1998), see http://www.ec-securehost. com/SIAM/SE06.html.

${ }^{61}$ In this case degeneracy can be "accidental," i.e., non-induced by symmetry. 